Therefore the respective approximate values for $x$ are:

$$
\begin{array}{rr}
.82573+.40364 i & -.82573-.40364 i \\
.28709+1.04944 i & -.28709-1.04944 i \\
-.28709+1.04944 i & .28709-1.04944 i \\
-.82573+.40364 i & .82573-.40364 i
\end{array}
$$

\title{
NOTE ON THE CIRCLE THEOREM OF HYDRODYNAMICS*
}

\section{By E. LEVIN (National Bureau of Standards)}

The circle theorem [1,2] is concerned with the irrotational, two dimensional flow of an incompressible, inviscid fluid in the $z$ plane. Let $f(z)$ be the complex potential of flow. Then if there are no singularities within a radius, $a$, of the origin and no rigid boundaries in the plane, the appropriate flow function after introducing a circle $|z|=a$ about the origin is given by $g(z)=f(z)+f^{*}\left(a^{2} / z\right)$ (where ${ }^{*}$ denotes conjugation).

The purpose of this note is to show that the restriction on rigid boundaries may be somewhat relaxed.

That some restriction is necessary may be seen from the following example. Consider the uniform flow $g(w)=w$. From the circle theorem, $h(w)=w+1 / w$ represents the flow with a unit circle about the origin. By the transformation $z=w+3 i$, we see that $H(z)=z-3 i+(z-3 i)^{-1}$ represents the flow about the circle centered at $3 i$. Then if a second unit circle is introduced about the origin, $F(z)=z+(z-3 i)^{-1}+z^{-1}+\left(z^{-1}+\right.$ $3 i)^{-1}$ should give the flow for the two circles. It can easily be verified that while $z=e^{i \theta}$ is a streamline, $z=e^{i \theta}+3 i$ is not.

A rigid boundary will be called generally admissible if for every flow $f(z)$ having this boundary as a streamline and satisfying the remaining conditions of the circle theorem, the function $g(z)=f(z)+f^{*}(1 / z)$ has both the unit circle and the boundary as streamlines. The boundary will be called conditionally admissible if this is true only for certain complex potentials $f(z)$.

To determine a necessary and sufficient condition for rigid boundaries to be generally admissible, consider any complex potential $f(z)=\alpha(x, y)+i \beta(x, y)$. Then

$$
\begin{gathered}
{[f(z)]^{*}=\alpha(x, y)-i \beta(x, y)=f^{*}\left(z^{*}\right)=f^{*}(x-i y),} \\
f^{*}(1 / z)=f^{*}\left(\frac{x}{x^{2}+y^{2}}-\frac{i y}{x^{2}+y^{2}}\right)=\alpha\left(\frac{x}{x^{2}+y^{2}}, \frac{y}{x^{2}+y^{2}}\right)-i \beta\left(\frac{x}{x^{2}} \frac{x}{2}, \frac{y}{x^{2}+y^{2}}\right), \\
g[g(z)]=g\left[f(z)+f^{*}(1 / z)\right]=\beta(x, y)-\beta\left(\frac{x}{x^{2}+y^{2}}, \frac{y}{x^{2}+y^{2}}\right) .
\end{gathered}
$$

Clearly if $x^{2}+y^{2}=1, g[g(z)]=0$, and hence the boundary of the unit circle is a streamline. Suppose $C: x=x(\tau), y=y(\tau), \tau_{0} \leq \tau \leq \tau_{1}$, is a rigid boundary. Then $g[f(z)]=$ $\beta[x(\tau), y(\tau)]=$ constant for $\tau_{0} \leq \tau \leq \tau_{1}$. In order that this rigid boundary be admissible, it is necessary and sufficient that $\mathscr{g}[g(z)]$ be constant along $C$. Hence $\beta\left[x /\left(x^{2}+y^{2}\right)\right.$,

${ }^{*}$ Received November 27, 1953. The preparation of this paper was sponso red (in part) by the Office of Naval Research, USN. 
$\left.y /\left(x^{2}+y^{2}\right)\right]=$ constant along $C$. That is, the inversion of the boundary with respect to the circle must also be a streamline of the original flow $f(z)$. To be generally admissible this inversion of the bqundary must be a streamline for every flow hence must itself be a rigid boundary. Thus a necessary and sufficient condition that rigid boundaries be generally admissible is that they be mapped into themselves under inversion with respect to the circle. A given boundary can be made conditionally admissible by obtaining the flow for this boundary together with its inversion and then regarding the inversion as only a streamline and not a boundary.

In particular, any plane barrier along $y=c x, a \leq x \leq b$, is generally admissible subject only to the condition that $a b=\left(1+c^{2}\right)^{-1}$. As an example of the application of the circle theorem under this modified restriction on rigid boundaries, consider

$$
f(z)=\frac{2}{3} \cos \theta[4 z-5]-i \frac{8}{3} \sin \theta\left[(z-2)\left(z-\frac{1}{2}\right)\right]^{1 / 2}
$$

representing flow at an angle $\theta$ about the plane boundary $1 / 2 \leq x \leq 2$. Then under the transformation $g(z)=f(z)+f^{*}(1 / z)$ it can be shown that both the unit circle and the plane boundary are streamlines. Any portion of this plane boundary is conditionally admissible with respect to this complex potential $f(z)$.

Acknowledgment. The author should like to express his gratitude to Professor R. Redheffer for helpful suggestions and criticism.

\section{REFERENCES}

1. L. M. Milne-Thomson, Theoretical hydrodynamics, 2nd ed., Macmillan and Co., London, 1949, p. 149.

2. L. M. Milne-Thomson, Hydrodynamical images, Proc. Camb. Phil. Soc. 36, 246-247 (1940).

\section{A NOTE ON A PAPER BY G. C. McVITTIE*}

\section{By G. B. WHITHAM (The University of Manchester)}

In a recent paper** in the Quarterly of Applied Mathematics G. C. McVittie uses Einstein's equations in general relativity to derive certain solutions of the classical equations of continuity and momentum for the compressible flow of a fluid when heat conduction and viscosity are neglected. Explicit expressions (which always satisfy these equations) are obtained for the density $\rho$, pressure $p$ and velocity components $U_{i}$ in terms of arbitrary functions $\phi, \phi_{1}, \phi_{2}, \phi_{3}$ of the time $T$ and space coordinates $X_{i}$; the $\phi$ 's are not independent however and must satisfy certain (differential) consistency equations. The equations of continuity and momentum are (using the double suffix summation convention)

$$
\begin{gathered}
\frac{\partial \rho}{\partial T}+\frac{\partial}{\partial X_{i}}\left(\rho U_{i}\right)=0 \\
\rho\left(\frac{\partial U_{i}}{\partial T}+U_{i} \frac{\partial U_{i}}{\partial X_{i}}\right)+\frac{\partial p}{\partial X_{i}}=0
\end{gathered}
$$

*Received December 2, 1953.

**G. C. McVittie, "A method of solution of the equations of classical gas dynamics, using Einstein's equations", Quart. of Appl. Math., 11, No. 3 (1953). 